\title{
Movement of a relativistic particle in a spherical potential box
}

\section{Yurasov N.I., Yurasova I.I.}

Bauman Moscow State Technical University, Moscow, Russia;

E-mail: Yurasov<nikyurasov@yandex.ru>;

The problem of a relativistic particle in a spherical potential box with impenetrable walls has investigated. The power spectrum has found. Comparison with the specified characteristics for a similar not relativistic problem has executed. Essential distinctions have revealed. Possibilities of application of the solved problem in astrophysics are considered.

Keywords: potential box, relativistic particle, a power spectrum, black hole, maximon, temperature of maximons. DOI: $10.18698 / 2309-7604-2015-1-559-565$

\section{Introduction}

Potential boxes make a basis of many microscopic models of physical objects. As many physical objects have three spatial measurements, but special interest represent 3-D potential boxes. Among such models the greatest symmetry the box spherical forms possesses. Such box is the natural candidate for modelling of a black hole. With various potentials and construction and the analysis of various models of black holes many works [1-11] are devoted questions of the analysis of this model and this direction intensively develops. The purpose of our work is the analysis of a spectrum of microparticles when their movement is relativistic and application of the received results to model of a black hole.

\section{The Klein-Gordon equation and power spectrum}

We use the Klein-Gordon equation [1]. As in [2] we investigate the symmetric decision. We enter dimensionless time and dimensionless radial coordinate by means of following formulas

$$
\tau=\frac{E_{R} t}{\hbar}, \quad \xi=\frac{r}{R},
$$

where $E_{R}=\frac{\hbar c}{R}, E_{R}-$ characteristic energy, $2 \pi \hbar=h$-constant Planck, $t-$ time, $r-$ radial coordinate, $R$-radius of a spherical potential box. Then the Klein-Gordon equation accepts the following initial form 


$$
\left(\frac{\partial^{2}}{\partial \tau^{2}}-\frac{\partial^{2}}{\partial r^{2}}-\frac{2}{\xi} \frac{\partial}{\partial \xi}+\varepsilon^{2}\right) \Psi=0
$$

where $\varepsilon=\frac{m c^{2}}{E_{R}}, \mathrm{~m}-$ mass of a particle in own system of readout.

The decision of the equation (2) on the Furie's method looks like

$$
\Psi=\mathrm{X}(\xi) \mathrm{T}(t)
$$

Function $\mathrm{X}(\xi)$ has been set in the form corresponding to not relativistic variant of a similar problem, namely

$$
\mathrm{X}_{n}(\xi)=A \frac{\sin (\pi n \xi)}{\xi}, n=1,2,3, \ldots
$$

where $A$ is constant and $n$ is number of a quantum condition.

This function automatically satisfies to a boundary condition for potential box

$$
\mathrm{X}_{n}(\xi=1)=0, n=1,2,3, \ldots
$$

Substituting (4) in (3) and (3) in (2) we receive the equation for function $\mathrm{T}_{n}, n=1,2,3, \ldots$

$$
\left(\frac{\partial^{2}}{\partial \tau^{2}}+\varepsilon^{2}+(\pi n)^{2}\right) \mathrm{T}_{n}=0
$$

The decision of the equation (6) looks like

$$
\mathrm{T}_{n}(\tau)=C_{1 n} e^{\lambda_{1 n} \tau}+C_{2 n} e^{\lambda_{2 n} \tau}, n=1,2,3, \ldots
$$

where $C_{j n}$ are constants, $\lambda_{j n}=(-1)^{j} i\left(\varepsilon^{2}+(\pi n)^{2}\right)^{1 / 2} ; j=1,2 ; n=1,2,3, \ldots$. Therefore the power spectrum of a particle in spherical potential box is defined by the formula 


$$
E_{n}= \pm E_{R}\left(\varepsilon^{2}+(\pi n)^{2}\right)^{1 / 2}, n=1,2,3, \ldots
$$

In a relativistic spectrum there are two branches corresponding to particles (+) and antiparticles (-).

\section{Not relativistic case}

Consider a symmetrical potential box of spherical form. Then the Shredinger's equation [1] looks like

$$
\left(i \frac{\partial}{\partial \tau}+\varepsilon_{R}\left(\frac{\partial^{2}}{\partial \xi^{2}}+\frac{2}{\xi} \frac{\partial}{\partial \xi}\right)\right) \Psi=0
$$

where $\varepsilon_{R}=\frac{\hbar}{2 c m R}$.

Under the same boundary condition the equation (9) has the decision

$$
\Psi_{n}(\xi)=A \frac{\sin (\pi n \xi)}{\xi} \mathrm{T}_{n N R}(\tau), n=1,2,3, \ldots
$$

where there is a decision of a following equation

$$
\left(i \frac{\partial}{\partial \tau}-(\pi n)^{2} \varepsilon_{R}\right) \mathrm{T}_{n N R}=0
$$

and the power spectrum is defined by the formula

$$
\mathrm{E}_{n}=\mathrm{E}_{R} \varepsilon_{R} n^{2}=\frac{(\pi \hbar n)^{2}}{2 m R^{2}}, n=1,2,3, \ldots
$$


Compare power spectra (8) and (12). At big numbers the relativistic power spectrum aspires to linear dependence on level number, and not relativistic power spectrum remains squarelaw. .

\section{Power spectrum of a particle in a box and substance of a black hole}

Consider the appendix of the executed calculations to the analysis of a condition of substance in a black hole. As properties of area in a black hole are known hypothetically and possible the various points of view, suppose, that black hole there is a potential box with impenetrable walls and it has the sphere form.

For radius of a black hole we use formula Laplas-Shvartzshilda [3]

$$
R=\frac{2 G m_{\otimes}}{c^{2}}
$$

where $\mathrm{m}_{\otimes}$ is a mass of a black hole, $\mathrm{G}$ is a gravitational constant.

Entering radius of a black hole (13) in formulas for power spectra (8), (12), we receive

$$
\begin{aligned}
& E_{n}= \pm\left(\left(m c^{2}\right)^{2}+\left(\frac{\pi \hbar c^{3} n}{2 G m_{\otimes}}\right)^{2}\right)^{1 / 2}, n=1,2,3, \ldots \\
& E_{n}=E_{R} \varepsilon_{R} n^{2}=\left(\frac{\pi^{2}}{8 m}\right)\left(\frac{\hbar c^{2} n}{m_{\otimes}}\right)^{2}, n=1,2,3, \ldots
\end{aligned}
$$

In the resulted calculations it has supposed, that in a potential box force fields were absent. If in a black hole there are force fields (not including of surface) the problem can be considered a method of the theory of indignations.

We yet did not mention a question on mass of particles moving in potential box. For a case of a black hole it is possible to assume equality of mass of such particle to mass of maximon on Markov or the Plank's mass $\mathrm{m}_{\mathrm{Pl}}$. Then own energy of this particle is equal

$$
\mathrm{E}_{0}=m_{P I} c^{2}=\left(\frac{\hbar c^{5}}{G}\right)^{1 / 2},
$$


and the formula for a power spectrum accepts the following form:

$$
\mathrm{E}_{n}=\left(\frac{\hbar c^{5}}{G}\right)^{1 / 2}\left(1+\frac{\pi^{2}}{4}\left(\frac{m_{P I}}{m_{\otimes}}\right)^{2} n^{2}\right)^{1 / 2},
$$

Let's estimate the second composed in brackets. As the relation of mass of a black hole to mass of the sun more than four [3,4], and mass of the sun about $\mathrm{m}=2 * 10^{30} \mathrm{~kg}[4]$ the mass of a black hole satisfies an inequality $\mathrm{m}_{\otimes}>8 * 10^{30} \mathrm{~kg}$. Considering mass maximon $\left(\mathrm{m}_{\mathrm{Pl}}=2 * 10^{-8} \mathrm{~kg}\right)$ we receive, that the estimated composed has an order of a square of the relation of mass of maximon to mass of a black hole. As a result both composed become sizes of one order under a condition

$$
n=\frac{2}{\pi} \frac{m_{\otimes}}{m_{P I}} \rightarrow n \geq 3 * 10^{38}
$$

On the other hand it is possible to estimate number of maximons, containing in a black hole. As an estimation it is used the simple formula

$$
N_{\max }=\frac{m_{\otimes}}{m_{P l}}
$$

Applying the formula (19), we receive

$$
N_{\max } \geq 4 * 10^{38}
$$

In a black hole it is a lot of maximons. Therefore it is possible to enter temperature of maximons. 


\section{Energy and temperature of particles in a black hole}

We will propose that the maximons temperature is great enough and it is possible to use the Boltzman's distribution. Therefore for energy of system of maximons we have the formula

$$
\mathrm{E}=N_{\max } \sum P_{n} \mathrm{E}_{n}
$$

where $P_{n}=\frac{\exp \left(-E_{n} / K T\right)}{\sum \exp \left(-E_{n} / K T\right)}$ is weight of condition, $K$ is the Boltzman's constant, $T$ is an absolute temperature. The formula (21) allows to receive the equation for temperature of maximons and, hence, the formula for temperature in a black hole. We will pass to reception of this formula. Equating average energy of thermal movement of gas and average energy from distribution Больцмана, we receive the required formula

$$
\begin{gathered}
\mathrm{T}-\frac{2}{3} \frac{\mathrm{E}_{P I}}{\mathrm{~K}} \frac{\sum f(n) \exp (-\beta f(n))}{\sum \exp (-\beta f(n))}=0, \\
\text { where } f(n)=\left(1+\frac{\pi^{2}}{4}\left(\frac{m_{P I}}{m_{\otimes}}\right)^{2} n^{2}, \beta=\frac{\mathrm{E}_{P I}}{\mathrm{KT}}, n=1,2,3, \ldots \infty .\right.
\end{gathered}
$$

From the formula (22) follows that the temperature of maximon gas depends on mass of a black hole. The temperature is essential parametre for research of thermal processes in a black hole, including possibility of evaporation of the black holes, for the first time considered by Howking and gravitation waves [8-9], and also a problem of stability of black holes [5-7,10-11].

\section{Conclusion}

The spectrum of maximonsin the spherical potential box, having radius of a black hole is received. The model of the black hole filled maximons is developed. With use of discrete distribution of Boltzman the equation defining temperature of maximons in a black hole is deduced. It is shown, that this temperature depends on weight of a black hole. 


\section{References}

1. Dyson F. (2007). Advanced quantum mechanics. Singapore: World Scientific Co. Ptc. Ltd.

2. Hassanabadi H., Yazartoo B.H., Zarrinkahar S. (2013). Exact solution of Klein-Gordon equation for Hua plus modified Eckart potentials. Few.-Body Syst., Vol. 54, N 11, 2017-2025.

3. Landau L.D., Lifshitz E.M. (2006). Theory of field. Moscow: Fizmatlit.

4. Sperhake U. (2014). Numerical relativity. The role of black holes in gravitational wave physics, astrophysics and high-energy physics. Gen. Relativ. and Grav., Vol. 46, N 5, 1-23.

5. Perez D., Romen G.E., Perez-Bergliaffa S. (2014). An analysis of a regular black hole interior model. Int. J. Theor. Phys., Vol. 53, N 3, 734-753.

6. Taji M., Malekjani M. (2013). Interaction holographic politropic gas model of dark energy. Int. J. Theor. Phys., Vol. 52, N 10, 3405-3412.

7. Rodrigues M.E., Marques G.T. (2013), Thermodynamics of a class of non-asymptotical flat black holes in Einstein - Maxwell dilaton theory. Gen. Relativ. and Grav., Vol. 45, N 7, 1297 1311.

8. Woods R.C., Baker R.M.L., Li F., Stephenson G.V., Davis E.W., Beckwith A.W. (2011). A new theoretical technique for the measurement of high-frequency relic gravitational waves. $J$. of Mod. Phys, N 2, P. 498-51.

9. Xiao X. (2014). Detecting the change of Howking temperature with geometric phase. Int. J. Theor. Phys., Vol. 53, N 3, 1070-1077.

10. Pourdarvish A., Pourhassan B. (2013). Statistic mechanics of a new regular black hole. Int. J. Theor. Phys., Vol. 52, N 11, 3908-3914.

11. Hennig J., Neugebauer G. (2011). Non-existance of stationary two-black-hole configurations. The generate case. Gen. Relativ. and Grav., Vol. 43, N 11, 3139-3162. 